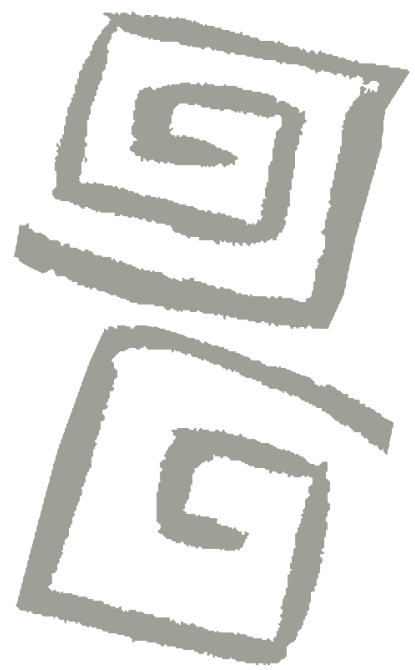

\title{
Crónica de un éxito consolidado: las políticas de erradicación de la viruela en Brasil (1962-1973)
}

\author{
Chronicle of a consolidated success: smallpox \\ eradication policies in Brazil (1962-1973)
}

Jonatan Sacramento ${ }^{1}$

'Estudiante de Doctorado, Programa de Pós-Graduación en Ciências Sociais, Instituto de Filosofia y Ciências Humanas, Universidade Estadual de Campinas, San Pablo, Brasil. $\bowtie$ iD
RESUMEN El objetivo de este artículo es comprender las acciones para combatir la viruela en Brasil, en el marco de la Campaña de Erradicación de la Viruela, que tuvo lugar en las décadas de 1960 y 1970. Se intenta argumentar que, además de la aguja bifurcada y de la vacuna liofilizada, la vigilancia epidemiológica -a partir de la construcción de instrumentos y protocolos de vigilancia en salud- fue el tercer elemento clave que garantizó la erradicación de la enfermedad. La hipótesis que se pretende sustentar es que las acciones de control y erradicación de la viruela contribuyeron a la construcción de nuevas instituciones de salud en el interior de Brasil. Como un ejercicio de análisis sociohistórico, la investigación se basó en fuentes documentales (informes, boletines y legislaciones), en entrevistas con profesionales directamente involucrados con la erradicación de la viruela y en parte de la producción intelectual de estos respecto del tema.

PALABRAS CLAVES Viruela; Erradicación; Vigilancia Epidemiológica; Políticas de Salud; Brasil.

\begin{abstract}
The aim of this article is to understand actions taken towards smallpox eradication in Brazil, in the framework of the Smallpox Eradication campaign that took place in the 1960s and 1970s. The article argues that, in addition to the bifurcated needle and the lyophilized vaccine, epidemiological surveillance - based on the construction of instruments and protocols for health surveillance - was the third key element that guaranteed the eradication of the disease. The hypothesis is that the actions taken towards the control and eradication of smallpox contributed to the construction of new health institutions within Brazil. As an exercise of socio-historical analysis, this research was based on documentary sources (reports, newsletters and legislation), interviews with professionals directly involved in the eradication of smallpox, and part of the intellectual production on the subject.
\end{abstract}

KEY WORDS Smallpox; Eradication; Epidemiological Surveillance; Health Policy; Brazil. 


\section{INTRODUCCIÓN}

Como crónica de un éxito consolidado, la erradicación de la viruela ocupa un lugar singular en la historia de la salud pública internacional. Sin embargo, en Brasil, los estudios sobre el tema parecen tener un lugar periférico en la historiografía de la salud pública, restringido a los análisis que enfatizan el importante episodio de la "revuelta de la vacuna", de 1904, cuando la población carioca de la naciente república se rebeló contra las medidas de obligatoriedad de la vacunación de la municipalidad de Río de Janeiro ${ }^{(1,2,3)}$. El interés por abordar la erradicación de la viruela es reciente $y$, hasta el momento, son pocos los trabajos que buscan rescatar la historia de la Campaña de Erradicación de la Viruela en el territorio brasileño ${ }^{(4,5,6,7,8)}$.

Sin embargo, observar la viruela y su campaña de erradicación permite comprender las relaciones entre la salud, la sociedad, el Estado y sus instituciones, además de los actores que conformaron tales experiencias. Como mostró Foucault( ${ }^{(9)}$, la respuesta estatal a las epidemias de viruela en la Europa del siglo XVIII provocó uno de los cambios político-organizacionales más relevantes en cuanto a la concepción de poder y su relación con los pueblos.

El surgimiento de la idea de población -como instancia que debería ser gestionada a través de un dispositivo de seguridad, como nueva técnica de poder- trajo aparejada la necesidad de combatir la viruela a fin de controlar las tasas de mortalidad, como consecuencia de los brotes de la enfermedad. Además, este dispositivo de seguridad permitió, a partir de la instauración de las estadísticas vitales y de los cálculos de probabilidad sobre expectativas de vida, no solo un control directo de la salud de la población, sino también su protección y un tipo menos peligroso de la enfermedad.

Según Foucault, la viruela pasó a ser entendida como un fenómeno colectivo, por lo que se insertó en la dinámica social del dispositivo de seguridad que nacía por entonces, dirigido a la demografía de la enfermedad y su control. Así, la noción de población nació como un correlato del poder y como objeto del saber a partir del control de la viruela en el siglo XVIIII' ${ }^{(9)}$.

$\mathrm{Si}$ el combate contra la viruela en aquel siglo sirvió para conformar la nueva forma del Estado y del poder estatal, en el Brasil de inicios del siglo XX, la lucha contra la viruela tuvo el mismo papel al impulsar la creación de instituciones científicas para la producción de la vacuna y generar la necesidad de mantener un cuerpo de empleados públicos para el combate contra las epidemias. Como mostró Hochman ${ }^{(10)}$, la "era del saneamiento" posibilitó no solo el combate contra las principales enfermedades que asolaban el interior del país, sino también la construcción de toda una burocracia sanitaria estatal en las décadas de 1920 y 1930, que se consolidaría en las instituciones que aún hoy, cien años más tarde, operan en el país. Guardadas las debidas proporciones y el contexto histórico, con la erradicación de la viruela parece haber sucedido algo parecido. Como será posible verificar a lo largo del artículo, la lucha contra la viruela, a partir de 1965, posibilitó redefinir las instituciones de salud a nivel nacional, la producción de insumos inmunológicos y las estadísticas sanitarias, además de la organización de una nueva rutina sanitaria en lo referente a la notificación y control de enfermedades.

Las publicaciones brasileñas sobre la erradicación de la viruela han mostrado cómo tales acciones estuvieron incluidas en un escenario más amplio de discusión al interior de la salud pública internacional sobre la temática de la erradicación de las enfermedades ${ }^{(4,5,5,7,7)}$, en la que la vigilancia epidemiológica se constituyó, al menos en lo que respecta a la erradicación de la viruela, en una metodología aplicada al control de enfermedades $^{(4)}$. En este sentido, Gazeta ${ }^{(5)}$ afirma que la Campaña de Erradicación de la Viruela, de 1966, apareció como política de salud pública en un contexto de articulación y esfuerzos internacionales de control y erradicación de la enfermedad, coordinados por la Organización Mundial de la Salud (OMS). Así, según la autora ${ }^{(6)}$, la estructura en la que 
estaba asentada la Campaña era inversamente proporcional al cuadro de gravedad de la enfermedad en el país, donde ya predominaba su forma "benigna" como consecuencia de las acciones de control emprendidas en el período 1962-1965.

Es en esa dirección que Hochman ${ }^{(7)}$ muestra cómo tales cambios en el contexto nacional e internacional relacionados con la política de erradicación y control de enfermedades se referían no solo a la salud pública, sino también a las cuestiones vinculadas al desarrollo económico y las relaciones internacionales entre las principales potencias políticas y económicas de la época, sobre todo EEUU y la Unión Soviética, entre otros. En el caso de Brasil, incluso sin que existiera un consenso entre los médicos y las autoridades nacionales sobre la importancia sanitaria de erradicar la viruela, tal hecho se presentaba como una oportunidad para crear una buena imagen del régimen militar de la época, además de haber contribuido a la construcción de una "cultura de inmunización" en el país ${ }^{(8)}$.

En este contexto, este artículo busca comprender el proceso de erradicación de la viruela en Brasil, sobre la base de la construcción de conocimientos epidemiológicos y la institucionalización de los aparatos sanitarios en el seno de las políticas de combate contra la viruela en el territorio nacional. Con el propósito de abordar las dinámicas de producción de saberes epidemiológicos y las políticas de salud pública, se busca mostrar cómo el combate contra la viruela y, principalmente, el redescubrimiento de la viruela como problema de salud pública, posibilitó la consolidación de un aparato sanitario, burocrático e intelectual de la salud pública brasileña, a través de la institucionalización de protocolos y prácticas sanitarias. La hipótesis que se pretende sustentar es que, más allá de la aguja bifurcada y de la vacuna liofilizada, indicadas por la bibliografía brasileña como esenciales para la erradicación, la vigilancia epidemiológica fue el tercer elemento clave para su consolidación.

Este artículo es parte de la investigación de maestría titulada Saberes, poderes e corporalidades: a biopolítica da erradicação da varíola, defendida en el marco del Programa de Posgrado en Antropología Social de la Universidade Estadual de Campinas en febrero de 2018. En la investigación, además del proceso de erradicación de la viruela, se buscó comprender las trayectorias intelectuales de los participantes en la lucha por la erradicación de la enfermedad y el contexto sanitario internacional en el que se inscribían tales acciones.

\section{METODOLOGÍA}

Desde un abordaje cualitativo, la investigación se basó en tres procedimientos: una revisión de la bibliografía nacional e internacional sobre la erradicación de la viruela en Brasil y en el mundo; análisis documental de los boletines epidemiológicos publicados por la Campaña de Erradicación de la Viruela entre 1967 y 1974, de la legislación nacional y estatal concerniente al tema, de las declaraciones de médicos y otros profesionales de salud que trabajaron en el ámbito de la Campaña, y de una entrevista con uno de los coordinadores de la Campaña.

\section{DEL ESCENARIO A LOS BASTIDORES: LA VIRUELA COMO PRIORIDAD SANITARIA NACIONAL}

En la historia de la salud pública brasileña, la viruela siguió la tríada prioridad, invisibilidad y erradicación $n^{(7,8)}$. Fue prioridad a inicios del siglo $\mathrm{XX}$, junto con la fiebre amarilla, al ser blanco de la campaña de vacunación obligatoria impuesta por el gobierno federal en las acciones dirigidas por el sanitarista Oswaldo Cruz (1872-1917) para la transformación de la ciudad de Rio de Janeiro durante el año 1904. Tal acción tenía como objetivo no solo sanear la capital de la joven república, librándola de la viruela y de la fiebre amarilla, sino también proporcionar un escenario de transformación urbanística en la configuración de la ciudad, transformación con claras marcas de raza y de clase ${ }^{(2)}$. 
En ese contexto de intervención pública, de desplazamiento poblacional e imposición de medidas profilácticas, que se sumaba al clima político de la República recién instaurada ${ }^{(1)}$, la población carioca se rebeló contra las acciones del poder público, a través de motines que duraron días y entraron en las páginas de la historiografía brasileña como la "revuelta de la vacuna". Momento crucial en la política brasileña de la época, la revuelta tuvo como consecuencias no solo la abolición de la prerrogativa de la obligatoriedad de la vacunación, sino la reestructuración de las acciones de salud pública en la capital brasileña, a partir de la definición de nuevas metas sanitarias y de la inauguración de un nuevo escenario para las medidas sanitarias referentes a la viruela y a la fiebre amarilla ${ }^{(11)}$.

Si bien con la interrupción de la obligatoriedad de la vacuna, la práctica de la vacunación continuó y contribuyó a la detención del número de casos de viruela en Brasil -al menos en los grandes centros urbanos- la enfermedad pasó a una fase de "invisibilidad" en las acciones de salud pública. La efectividad de la vacuna en el combate contra la viruela dio lugar a que el gobierno brasileño adoptara, como pauta prioritaria de las acciones de salud, la continuidad del combate contra la fiebre amarilla y las Ilamadas "enfermedades del sertón", tales como la anquilostomiasis, la difteria, la recién descubierta enfermedad de Chagas y la malaria ${ }^{(10,12)}$. La viruela solo regresaría a la escena de la salud pública nacional a partir de la década de 1960 .

\section{DE LOS BASTIDORES AL ESCENARIO: LA VIRUELA DE REGRESO A LA ESCENA SANITARIA NACIONAL}

La última fase de la viruela en las políticas públicas de salud en Brasil, la de la erradicación, comenzó recién en el año de 1962, con la Campaña Nacional Contra la Viruela, implementada por el entonces presidente João Goulart (1961-1964), y cuyo objetivo era aumentar la cobertura nacional de la vacunación contra la enfermedad, que ya no era considerada grave $\mathrm{e}^{(4,6)}$. Sin embargo, para que tales acciones se concretaran, la viruela tuvo que ser "redescubierta" como problema de salud pública. Aun sin un sistema de estadísticas sanitarias confiable, era de conocimiento de las autoridades brasileñas que en el país la viruela mayor, forma más grave de la enfermedad, convivía con la menor, forma benigna y no letal, y que el escenario de grandes brotes de la enfermedad ya no era común, como había sido hasta comienzos del siglo $X X$. En ese contexto, en sintonía con las discusiones internacionales, el control de una enfermedad contagiosa como la viruela era visto como señal de desarrollo económico y social, por lo tanto, debería entrar en la agenda del proceso desarrollista emprendido por el entonces presidente João Goulart ${ }^{(6)}$.

Cuatro años después, en 1966, durante la Asamblea Mundial de la Salud de la Organización Mundial de la Salud (OMS), cuando fue aprobada la erradicación de la viruela como meta a ser alcanzada por los países miembros, Brasil decretó el inicio de las actividades de la Campaña de Erradicación de la Viruela, por ser el único país del continente americano que no había erradicado la enfermedad.

Fue al interior de las dinámicas de este contexto social donde la Campaña de Erradicación de la Viruela alcanzó su objetivo en 1971, año del registro del último caso de viruela en el territorio brasileño, ocurrido en la ciudad de Río de Janeiro ${ }^{(13,14)}$. El certificado de erradicación de la viruela en Brasil fue oficialmente emitido por la OMS en 1973. Siete años después, en 1980, se publicó la Resolución No. 55 del Ministerio de Salud, que puso fin a la vacunación contra la viruela ${ }^{(15)}$. La erradicación mundial de la enfermedad, hecho que también sucedió gracias a la colaboración de los especialistas brasileños y a partir de la experiencia exitosa desarrollada en el país, se dio en 1977, luego del registro, en Somalia, del último caso de viruela; $y$, en 1980, la Comisión de Erradicación de la Viruela de la OMS emitió el certificado mundial de erradicación de la enfermedad ${ }^{(16)}$. 
La Campaña Nacional Contra la Viruela (1962-1965)

Creada en 1962, en el gobierno del presidente João Goulart (1961-1964), la Campaña Nacional Contra la Viruela fue una iniciativa del Gobierno Federal que apuntaba a controlar la incidencia de la viruela en el territorio nacional e impedir el surgimiento de nuevas epidemias de la enfermedad ${ }^{(17)}$. En aquel momento, todavía convivían la viruela mayor, forma más grave de la enfermedad y que podía causar la muerte o dejar secuelas como la ceguera, y la viruela menor, forma benigna de la enfermedad y con tasa de mortalidad casi cero.

Si bien fue creada en el ámbito nacional, la implementación y la operación de las actividades de la Campaña Nacional Contra la Viruela se daban de forma descentralizada en el ámbito estatal y municipal, y quedaba bajo la responsabilidad de la Unión solo la planificación de las acciones, la uniformización de las prácticas y el control de los datos epidemiológicos de la acción en sí( ${ }^{(7)}$. De esa forma, como cada estado, municipio y territorio eran responsables de organizar y poner en práctica la vacunación contra la viruela, el control de la enfermedad proveniente de las acciones de la Campaña Nacional Contra la Viruela no fue regular. Al contrario, las tasas de cobertura de vacunación, aunque hayan contribuido al control de la enfermedad, variaban de región a región y estaban sujetas al tipo de prioridad que la viruela ocupaba en la agenda de salud de cada localidad ${ }^{(7)}$. Tales esfuerzos, aunque hayan contribuido a la disminución de las tasas infección y también a la disminución de la forma mayor de la viruela, no fueron suficientes para erradicar o incluso eliminar la enfermedad ${ }^{(18)}$. Así, en la década de 1960, Brasil era el único país del continente con viruela.

En 1966, en el gobierno militar del General Castelo Branco, se firmó el decreto que instituyó la Campaña de Erradicación de la Viruela (Campaña), cuya meta era erradicar la enfermedad en el país. Integrada a un contexto de esfuerzos internacionales más amplios de erradicación de la enfermedad ${ }^{(19)}$, la Campaña fue implementada con el soporte administrativo, intelectual y financiero de la Organización Panamericana de la Salud (OPS), brazo americano de la Organización Mundial de la Salud (OMS), luego que la Asamblea Mundial de la Salud de 1965 decidió la erradicación mundial de la viruela. En ese contexto, cabe aclarar algunas posiciones en el escenario sanitario y político internacional de la época.

En el plano internacional, la trayectoria de la erradicación de la enfermedad se remonta a los inicios de la década de 1950, cuando Fred Soper (1893-1977), entonces director de la OPS (1947-1959) y entusiasta de las erradicaciones, le propuso al organismo la erradicación de la viruela en el continente americano. La propuesta fue acatada e incluso algunos países implementaron iniciativas en esa dirección sin tener presupuesto de la OPS para tales acciones. Hasta 1966, el único país del continente americano que no había erradicado la viruela era $\mathrm{Brasil}^{(20)}$, en tanto que Cuba, EEUU, México, Perú y Chile habían erradicado la enfermedad en 1923, 1949, 1952, 1957 y 1959, respectivamente, en un período anterior a la iniciativa de la OMS, en el contexto de esfuerzos nacionales de combate y control de las epidemias. Según Donald A. Henderson (1928-2016), director del programa de erradicación de la viruela de la OMS, el motivo que habría llevado a la Unión de las Repúblicas Socialistas Soviéticas (URSS) a proponer la erradicación de la enfermedad, en la Asamblea Mundial de la Salud de 1958, sin ser acatada por el organismo, era el hecho de que los países soviéticos en Asia sufrían los casos de viruela exportados por los países vecinos ${ }^{(20)}$. Además, la URSS era, en aquella época, el país con mayor producción de vacuna antivariólica y una de las mayores autoridades sobre el tema.

En el ámbito mundial, principalmente en las acciones de erradicación de los países de África, la United States Agency for International Development (USAID) aportaría los recursos logísticos para actuar y el Centers for Disease Control and Prevention (CDC), el personal técnico y la experticia epidemiológica. Cada país que desarrollara el programa 
sería responsable de adquirir las vacunas; sin embargo, aunque costaban menos de un dólar, no estaban en sus listas de prioridades en salud pública -al contrario de lo que sucedía con la fiebre amarilla- por lo que se adquirían a través de una política de préstamos de EEUU a los países interesados.

La estrategia del CDC para la viruela era simple y se basaba en dos acciones: la vacunación preventiva, a fin de reducir el número de personas susceptibles, y la creación de sistemas de vigilancia epidemiológica, que tenían como objetivo monitorear los casos de la enfermedad ${ }^{(20)}$. En los lugares donde la red básica de salud fuera insuficiente, se debía vacunar masivamente, de manera de conseguir, como mínimo, el $80 \%$ de cobertura de vacunación. La adopción de la estrategia de vigilancia y de control, a su vez, se apoyaba en la necesidad de recolectar datos sobre la epidemia pues, en muchos países, las estadísticas sobre la enfermedad eran inexistentes.

En 1967, el Dr. William Bill Foege implementó ciertas medidas en Nigeria que se tornaron la estrategia oficial de la OMS en la lucha contra la viruela: la vacunación vía vigilancia epidemiológica y de bloqueo, y ya no a través de la vacunación masiva que se realizaba hasta entonces, lo que contradecía la creencia sobre la necesidad de vacunar a toda la población mundial para acabar con la viruela ${ }^{(21)}$. En ese mismo momento, a pesar de la gran inversión de EEUU, a través de la USAID y del CDC, la URSS detentaba el protagonismo como mayor entusiasta de la campaña, apoyando la acción de Henderson como coordinador general y siendo uno de los mayores donadores de vacuna liofilizada usadas en los procedimientos de erradicación ${ }^{(22,23,24)}$.

En Brasil, al contrario de lo que sucedió en países de África y de Asia y en la In$\mathrm{dia}^{(4,5,6,7,7,8,20)}$, la estrategia oficial adoptada por la Campaña fue la de vacunación masiva y no de vigilancia epidemiológica. Los coordinadores de la Campaña, Oswaldo Silva $y$, posteriormente, Claudio Amaral optaron por el uso de la vacuna liofilizada, sumada a los inyectores a presión (Ped-O-Jet) y a la táctica de vacunación masiva en espacios públicos ${ }^{(13,14)}$. Sin embargo, como mostraré a continuación, el modo en que la Campaña de Erradicación de la Viruela se organizó en Brasil posibilitó la experimentación de otros métodos y técnicas y la creación de una experticia en salud pública, que serían posteriormente exportadas y adaptadas al combate contra otras enfermedades.

\section{La Campaña de Erradicación de la Viruela (1966-1971)}

Creada por el Decreto Ley 59153, de 1966, la Campaña de Erradicación de la Viruela fue una acción de salud pública coordinada por el Ministerio de Salud, a través de la Fundación Servicio Especial de Salud Pública (SESP), órgano federal especializado en grandes acciones de salud pública, que operó en Brasil durante el período 1942-1990 junto con la Campaña de Erradicación de la Malaria y la colaboración internacional de la OPS/OMS ${ }^{(25)}$. El objetivo de la Campaña era erradicar la viruela en territorio nacional a partir de la vacunación de, como mínimo, el $90 \%$ de la población. Estructurada en tres partes, preparación, ataque y mantenimiento, la Campaña contaba con recursos propios, oriundos del Ministerio de Salud y con apoyo financiero y técnico proveniente de organismos internacionales.

El decreto imponía la figura de un superintendente para la coordinación nacional de la Campaña. El profesional indicado por el director general del Departamento Nacional de Salud del Ministerio de Salud, elegido por ser un "técnico de reconocida competencia”(25), tenía entre sus funciones las tareas de orientar, coordinar y fiscalizar los trabajos de la Campaña a partir de las directrices propuestas por el plan anual elaborado por el propio superintendente. Además, las resoluciones vinculadas a la admisión y despido de personas involucradas en la Campaña y a la administración de los recursos estarían bajo su responsabilidad. A este profesional le correspondía también la recomendación de asesores y coordinadores regionales que tendrían a su cargo intermediar entre las 
secretarías de salud estatales y sus respectivas campañas y la Campaña nacional.

En cuanto a los recursos humanos, la Campaña incorporaría empleados del Ministerio de Salud que pudieran ser transferidos a las actividades de erradicación, así como otros empleados federales, o de los niveles estatales y municipales, liberados de sus funciones con esa finalidad. Además, la Campaña contaba con la prerrogativa de libertad de contratación de profesionales propios para las acciones que serían desarrolladas, tanto las de vacunación como las de orden técnico ${ }^{(25)}$.

Si en el plano de la ley, la estructura organizacional de la Campaña podía parecer anémica, en el cotidiano de sus acciones, la realidad era otra. La Fundación SESP, órgano especialista en salud pública y con experiencia en acciones de enfrentamiento de epidemias y endemias, proporcionó los médicos y los técnicos necesarios para la consolidación de las acciones de la Campaña. Al mismo tiempo, con recursos oriundos de la OPAS/ OMS, la Campaña pudo contar con consultores nacionales e internacionales, especialistas en salud pública y enfermedades epidémicas, que compartían su experticia para contribuir a que la Campaña lograra sus objetivos. Además, los estados de la federación tenían autonomía para montar sus campañas, siempre y cuando siguieran las directrices básicas impuestas por la Campaña nacional. Eso llevó, a su vez, a la creación de algunas estrategias no previstas en los planes iniciales, como la experiencia de la vigilancia epidemiológica en los estados de Paraná, Rio Grande do Sul y Bahia, por ejemplo ${ }^{(26,27)}$.

La fase de preparación antecedía el inicio de las actividades de vacunación y apuntaba al reconocimiento de las áreas donde se realizaría la Campaña, a fin de elaborar las estrategias de acción, además del entrenamiento del personal de campo y las actividades de educación sanitaria junto a la población. Luego seguía la fase de ataque, que iniciaba sus actividades después de definidas las estrategias de vacunación. En esa etapa, existía cierta flexibilidad con las normativas nacionales en lo concerniente a las acciones desarrolladas localmente, pero había un protocolo mínimo a seguir que solo podría ser adaptado en casos excepcionales. En esa fase existían a su vez dos momentos de evaluación: el diario y el semanal, en los que se evaluaban no solo los trabajos realizados, sino también, la efectividad y el alcance de la vacunación. La última fase era la de mantenimiento. Su objetivo era organizar un sistema de notificación de casos de viruela, a los fines del control epidemiológico, tarea que era responsabilidad de los servicios locales (estatales y municipales), y que realizaban los profesionales de salud y la comunidad, principalmente, en zonas con deficiencia de servicios de salud. Además, esa fase preveía la vacunación del $90 \%$ de los nacidos vivos en determinado año y la revacunación anual del $20 \%$ de la población.

Una vez finalizada la fase de ataque, se instalaba en cada estado la unidad de vigilancia epidemiológica, las que se descentralizaban en puestos de notificación municipales y/o regionales, de modo que, en algunos municipios, podía haber más de un puesto de notificación. La función del puesto de notificación era la vigilancia epidemiológica de posibles casos de viruela, la investigación de casos sospechados, la realización del diagnóstico, incluso con la colecta de material purulento, la derivación a los laboratorios acreditados para hacer el examen, y la notificación de estos casos a la unidad de vigilancia epidemiológica del estado la que, a su vez, debía compilar los datos informados por los puestos de todo el estado y enviarlos a la Campaña nacional.

La estrategia general de la Campaña nacional se asentaba en la vacunación masiva y casa por casa, por medio de la utilización de la aguja bifurcada, fácil de ser transportada y manipulada y que podía ser reutilizada en los días siguientes. En las acciones de vacunación masiva, la estrategia era reunir un conjunto de personas en espacios y eventos públicos, tales como plazas, terminales de ómnibus o festividades. En esas ocasiones, el instrumento usado era el inyector a presión, que permitía la vacunación de aproximadamente 1.000 personas por hora. Así, en pocas horas, dependiendo del número de 
habitantes de la ciudad, la Campaña conseguía cumplir la meta de vacunación del $90 \%$.

Sin embargo, la estrategia de puestos itinerantes ubicados en puntos de intenso movimiento en las ciudades no siempre tuvo el efecto deseado con relación al público objetivo que, muchas veces, estaba de paso en la ciudad y no disponía de tiempo para la vacunación $^{(14)}$. En ese sentido, valía recurrir a las escuelas, radios locales, ferias e iglesias, de modo de divulgar la acción de vacunación.

La Campaña nacional, instituida en 1966, tenía un calendario que definía el cronograma de inicio de las actividades en cada estado. Así, la vacunación comenzó por la región nordeste $y$, una vez finalizada la etapa de ataque en cada estado, "descendían" y se propagaban hacia otras regiones de Brasil. Al mismo tiempo, dado el carácter flexible de la Campaña, era posible que cada estado organizara su propia acción fuera del calendario nacional, como fue el caso de San Pablo y de Bahía, que iniciaron sus campañas en 1968 y con recursos propios.

En San Pablo, la Campaña se inició en $1967^{(28)}$ y comenzó a operar efectivamente en 1968. Como en su versión nacional, la Campaña de San Pablo también era comandada por un superintendente y estaba organizada en tres tipos de actividades: las administrativas, que comprendían la dirección, la comunicación, los recursos humanos, la logística de materiales, el financiamiento, el mantenimiento de transportes y la conserjería; las actividades técnicas, organizadas en epidemiología y estadística, educación sanitaria, entrenamiento y vacunación; y, por último, las actividades de campo, a cargo de jefes, asistentes, supervisores y vacunadores.

Al igual que en la Campaña nacional, el decreto paulista preveía fondos propios para sostener las acciones de la Campaña. En cuanto a la justificación para su creación, el decreto incluía doce ítems, de los cuales se destacan la notificación obligatoria de la viruela -resaltado como procedimiento patrón adoptado internacionalmente- y el argumento de que Brasil, en el período 19631965, contaba con el mayor porcentaje de notificación de casos, lo que representaba el
$85 \%$ de los registros generados en el continente americano ${ }^{(29)}$.

Otro argumento destacado era que la vacuna antivariólica de rutina se había mostrado ineficaz como medida profiláctica, sin ofrecer la protección esperada. Además, según señalaba el decreto, el estado de San Pablo recibía un gran contingente de inmigrantes de diversas partes del país, entre ellos, aquellos que serían portadores del virus de la viruela, lo que implicaba un factor de riesgo, dado que el $39 \%$ de la población del estado era susceptible a la enfermedad ${ }^{(28)}$.

Es importante señalar que el estado de San Pablo hacía tiempo que era un ejemplo en cuanto a las políticas públicas de salud. Desde el inicio del siglo XX, con las acciones de saneamiento rural que serían copiadas por el gobierno federal ${ }^{(10)}$, la tierra de los "bandeirantes" aparecía como ejemplo a ser seguido o como contrapunto a ser pensado en cuanto a la formulación y ejecución de medidas profilácticas.

Si bien en el ámbito nacional la fase de ataque de la Campaña era de responsabilidad del gobierno federal y la fase siguiente, la de mantenimiento, de los gobiernos locales, en San Pablo, el gobierno paulista asumió las tres fases como su responsabilidad. La fase de ataque paulista presuponía vacunar al $90 \%$ de la población y preveía finalizar en junio de 1970. En ese momento, mientras la Campaña nacional ya había comenzado la fase de consolidación, la campaña paulista tenía como objetivo evitar la propagación de la viruela a través mantener la vacunación de bloqueo, en caso de brotes o episodios aislados.

A los fines de organizar la fase de mantenimiento, que incluiría la vigilancia epidemiológica de la viruela, la Campaña de San Pablo contaba no solo con los puestos de notificación, sino también con la ayuda de los registros civiles, que remitían los datos para que la unidad de vigilancia epidemiológica realizara informes regulares sobre los nacidos y los registrados en las instituciones. De esa forma, la vigilancia epidemiológica conseguía tener un control de los nacidos y vacunados en todos los municipios. 
En San Pablo, el equipo de vigilancia epidemiológica estaba formado por un médico epidemiólogo, una enfermera de salud pública y una educadora sanitaria. Al médico le correspondía la supervisión general de las actividades, además de la responsabilidad de la vigilancia epidemiológica y la vacunación. La educadora sanitaria era responsable de la supervisión de los trabajos educativos, además de la formulación y aplicación de cursos y entrenamiento para médicos y personal auxiliar de los puestos de notificación ${ }^{(30)}$. La enfermera de salud pública debía supervisar las actividades de enfermería y trabajar junto a la educadora sanitaria para formular e impartir los programas de educación sanitaria y los entrenamientos ${ }^{(19,24,31)}$.

Sin embargo, no fue solo el estado de San Pablo que gozó de las prerrogativas de autonomía en la organización de las acciones de la Campaña. Los estados de Paraná, Rio Grande do Sul y Bahia, principalmente, tuvieron una actuación destacada en las acciones de vigilancia epidemiológica para el combate de la enfermedad, experiencias que fueron cruciales para decidir los nuevos rumbos de la erradicación de la viruela en Brasil y para la consolidación de un sistema nacional de vigilancia epidemiológica, la erradicación de la poliomielitis y, posteriormente, la exportación de la experticia de los epidemiólogos brasileños para actuar en la erradicación de la viruela en África y Asia.

En África, las acciones de erradicación de la viruela, incentivadas por la OMS y financiada por EEUU, estuvieron encuadradas en el Smallpox Eradication Program (SEP) lanzado por el gobierno estadounidense en el contexto de la Global Great Society ${ }^{(32,33)}$, creada por el presidente demócrata Lyndon B. Johnson (1908-1973) en 1964, y que tenía como objetivo apoyar el desarrollo económico y social de los países más pobres a partir de la expansión de una agenda liberal que incluía combatir el hambre, las enfermedades y el comunismo ${ }^{(33)}$. En el contexto de esas acciones, localizadas en medio del fuego cruzado de la Guerra Fría y de la disputa entre EEUU y la URSS, el SEP estadounidense tenía entre sus objetivos el control del sarampión en el continente africano, a partir de la venta de una nueva vacuna desarrollada en EEUU y que sería comprada por los países de África central y occidental a partir de préstamos otorgados por la USAID ${ }^{(20)}$.

En Asia, a su vez, principalmente en India, país que en 1962 albergaba más del $60 \%$ de los casos de viruela notificados en el mundo $^{(34,35)}$, la actuación de la campaña de la OMS se mostró más complicada. Para que la tarea de erradicación de la enfermedad fuese llevada a cabo, la OMS y su sucursal regional, la World Health Organization in South-East Asia (SEARO), tuvieron que juntar esfuerzos con el gobierno indio, tanto a nivel federal, negociando con el Poder Ejecutivo y el Ministerio de Salud, como a nivel local, lidiando con los estados y los líderes y burocracias de salud locales ${ }^{(12,34,36,37)}$. Al mismo tiempo, para que la empresa de la erradicación fuese exitosa, el plan estratégico de acción de los vacunadores y del equipo de combate contra la viruela tuvo que ser adaptado para dar cuenta de la realidad local, como en el caso del uso de la vigilancia epidemiológica en vez de la vacunación masiva, como mostraré más adelante ${ }^{(13,14)}$.

\section{La erradicación de la viruela y la estrategia brasileña de vigilancia epidemiológica}

Como ya fue señalado, la estrategia de vacunación vía vigilancia epidemiológica era una prerrogativa de la OMS para las campañas de erradicación de la viruela. Sin embargo, como también mostré, la Campaña de Brasil prefirió adoptar el método de vacunación masiva y, posteriormente, cuando concluyó la fase de ataque, implementó el control y la vigilancia epidemiológica, que tenían como objetivo contener los casos episódicos de la enfermedad. Sin embargo, en los estados de Paraná, Rio Grande do Sul y Bahia surgiría un grupo, ligado a la Fundación SESP, que estructuraría la Campaña estatal a partir de la estrategia de vigilancia y vacunación de bloqueo.

La idea de estructurar la erradicación en torno a un conjunto de datos epidemiológicos 
sobre los cuales planificar las acciones, metas y evaluaciones es antigua y fue utilizada desde la década de 1950, pero de forma aislada en casos de cuarentena ${ }^{(23)}$. El abandono de esa idea con la intención de abarcar una escala más amplia y colectiva, se dio en 1964, a partir de la producción del epidemiólogo checo, Karel Raska (1909-1987). En EEUU, el CDC fue el gran divulgador de este nuevo concepto, a partir de la producción de Alexander Langmuir (1910-1993), epidemiólogo de la University John Hopkins y creador del Epidemic Intelligence Service del órgano.

\section{La experiencia gaúcha y paranaense de vigilancia epidemiológica de la viruela}

En Brasil, la erradicación de la viruela vía vigilancia y control fue testeada, en primer lugar, en el estado de Paraná. En 1970, la Campaña estaba sin recursos económicos para iniciar las actividades de vacunación en el estado, hecho que llevó a la Fundación SESP, en el ámbito de la Campaña de Erradicación de la Viruela, a testear el método de vigilancia y control, en vez de la vacunación masiva. En aquel momento, Ciro de Quadros (1940-2014), médico oriundo de la Facultad de Medicina de la Santa Casa de Porto Alegre, asumió la función de organizar el Centro de Investigación Epidemiológica del estado con base en las acciones coordinadas por la Campaña. En ese contexto, en vez de la vacunación masiva, se investigaron los casos sospechosos de viruela notificados a la secretaría estatal. A partir de estos casos, la estrategia de vacunación se basaba en vacunar a toda la población de la región en la que se hubiese confirmado un caso sospechoso de viruela $^{(36,37)}$

La experiencia de Paraná en la erradicación, vía vigilancia y control, permitió descentralizar las acciones de combate contra la enfermedad $y$, al mismo tiempo que la coordinación nacional de la campaña se mantenía centralizada, estructuraba sus acciones de forma alineada con el objetivo de la Campaña nacional.
En Rio Grande do Sul, Airton Fischmann (1941) y Claudio Marcos da Silveira (1936), también formados por la Santa Casa de Porto Alegre, fueron contratados por la Secretaría Estatal de Salud para trabajar en la Unidad de Vigilancia Epidemiológica del estado, por un convenio instituido, en 1969, con la Fundación SESP. En aquella época, como fue dicho anteriormente, para la Campaña, la noción de vigilancia epidemiológica era un complemento de la vacunación masiva.

En tierras gaúchas, las acciones de erradicación de la viruela se dieron de un modo híbrido, uniendo la acción vía vacunación masiva y la vacunación vía vigilancia epidemiológica. A su vez, se caracterizaba por la colecta de datos de posibles casos de viruela, análisis preliminar $y$, en caso de confirmación, la vacunación de bloqueo en aquellas personas cercanas al enfermo y de los que se encontraban dentro de cierto radio de extensión. La Campaña de Rio Grande do Sul comenzó a trabajar en la ciudad de Porto Alegre y tenía planificado seguir hacia el interior del estado al finalizar la vacunación masiva de la capital. Sin embargo, Airton Fischmann y otros empleados de la Unidad de Vigilancia Epidemiológica ya estaban trabajando en la implementación de los puestos de vigilancia y notificación y, a partir de los datos recolectados por esos organismos, en la vacunación de bloqueo de los casos sospechosos ${ }^{(26,27)}$.

El modelo de vigilancia epidemiológica de la viruela, adoptado en Rio Grande do Sul, fue pensado por un grupo de médicos de la Fundación SESP cuya preocupación no solo era erradicar la viruela, sino también montar un sistema de vigilancia epidemiológica que pudiera ser utilizado para otras enfermeda$\operatorname{des}^{(27)}$. Además de erradicar la viruela y de implementar las unidades de vigilancia epidemiológica, la Fundación SESP tenía interés en la formación de recursos humanos en el área de salud, incluso en epidemiología. Así, a la par de la formulación de detalladas normas de recolección y tratamiento de los datos, de modo de garantizar su calidad técnica, la Fundación consiguió modernizar las estadísticas epidemiológicas a través de la informatización de esos datos ${ }^{(26,27)}$. 
A partir de la experiencia en la erradicación de la viruela, Rio Grande do Sul pudo institucionalizar la práctica de vigilancia epidemiológica, sobre todo por la incorporación de los auxiliares de epidemiología formados en la Fundación SESP y la inclusión de otras enfermedades para ser notificadas y combatidas, como fue el caso de la poliomielitis. Tal experiencia sería replicada más tarde, en el ámbito nacional, a través de la circulación de los profesionales gaúchos entre la Fundación SESP y el Ministerio de Salud ${ }^{(38)}$.

\section{La vigilancia epidemiológica desdoblada: el Boletim da Campanha de Erradicação da Varíola}

Como forma de consolidar la erradicación de la viruela, la Campaña instauró un mecanismo de control y colecta de información de casos sospechosos de viruela en todo el territorio nacional, no solo en los estados en los que la campaña no había sido iniciada, sino también en aquellos que ya estaban en la fase final de control y mantenimiento. Así, a partir de la notificación de casos sospechosos se comunicaría a la vigilancia epidemiológica de la viruela, para poder investigarlos y ejecutar la vacunación de bloqueo, si fuese necesario, y tener un panorama más claro de la efectividad de las acciones de la Campaña, con el propósito de evaluar el programa como un todo.

En ese sentido, en 1967, se creó el Boletim da Campanha de Erradicação da Varíola(39), publicación semanal que circuló hasta el año 1974, cuando fue sustituido por el Boletim Epidemiológico. La publicación estaba dividida en secciones y contenía no solo datos epidemiológicos sobre la erradicación de la viruela en el mundo (principalmente en América Latina), sino también materiales y entrevistas con especialistas en el tema; además de la sección de notificación semanal del número de casos de viruela en Brasil, de notificación obligatoria, y las notas epidemiológicas que traían noticias y datos sobre la erradicación de la enfermedad en otras partes del mundo.
En los boletines también aparecía la descripción de los principales síntomas de la viruela, con la intención de facilitar a los profesionales de la salud la identificación de un (posible) enfermo. Los síntomas eran dolor de cabeza intenso, dolores en la columna vertebral (raquialgia), malestar, escalofríos y temperaturas entre $39^{\circ}$ y $40^{\circ}$. Las pústulas, características de la viruela, aparecían a partir del sexto día y se manifestaban, principalmente, en el rostro, los brazos y las piernas. Dependiendo del estado de las pústulas y de los síntomas manifiestos en cada individuo, la viruela podría ser confundida con la varicela -también conocida en Brasil como cataporala sífilis o el herpes. En función de las características imprecisas de la viruela, el diagnóstico definitivo solo podía ser realizado mediante estudios de laboratorio, de allí la necesidad de popularizar los síntomas, de manera que los casos sospechosos pudieran ser registrados y se tomaran las medidas necesarias.

Las "Notas epidemiológicas", una de las secciones del Boletim, se dedicaba a hacer públicos los números de casos notificados por las direcciones regionales de salud, que se clasificaban como "viruela", "sin viruela" y "diagnóstico dudoso". Los exámenes de confirmación se realizaban en tres laboratorios: en San Pablo, en el Instituto Butantan; en Rio de Janeiro, en el Instituto Oswaldo Cruz (IOC/FIOCRUZ); y en Rio Grande do Sul, en el Laboratorio Central del Estado (LACEN).

Como se trataba de una enfermedad de notificación obligatoria, la sistematización de los datos epidemiológicos de viruela seguía el siguiente recorrido: desde los municipios hacia las direcciones regionales de salud; desde los estados hacia las secretarías estatales de salud; desde la Unión hacia el Ministerio de Salud, en especial, al Departamento Nacional de Salud. A su vez, el superintendente de la Campaña nacional enviaba a la OPS/OMS la información sobre el cuadro epidemiológico de la enfermedad en el país.

Además, se recolectaban datos sobre el cuadro epidemiológico de los estados brasileños con relación a otras enfermedades ya que, a comienzos de la década de 1970, la Campaña se alineó con el Gobierno Federal, 
en el ámbito del Ministerio de Salud, y con los gobiernos estatales y municipales, a través de las secretarías estatales y municipales de salud, con la finalidad de introducir otras vacunas en la campaña, práctica conocida como multivacunación, que consistía en suministrar más de una vacuna el mismo día ${ }^{(18)}$, a diferencia de la vacuna combinada que incluía antígenos para dos o más enfermedades, como la viruela y el sarampión. La multivacunación fue un procedimiento común, adoptado por la mayoría de las campañas estatales. Dada las condiciones, muchas veces adversas, encontradas por las autoridades sanitarias municipales, sumar las vacunas del sarampión, tétano y de otras enfermedades, a la vacunación obligatoria de la viruela, aumentaba el alcance de las acciones de profilaxis, incluso para grupos y regiones antes no atendidos por los servicios de salud comunes.

En 1974, cuando el Boletim da Campanha de Erradicação da Varíola fue reformulado y pasó a llamarse Boletim Epidemiológico, la publicación comenzó a publicar datos relativos a otras enfermedades que cobraron estatus de notificación obligatoria, tales como el sarampión y la poliomielitis, segunda enfermedad a ser erradicada en Brasil y blanco de una campaña internacional. Un año después, en octubre de 1975, fue publicada la Ley 6259 , que regulaba la organización de la vigilancia epidemiológica en el ámbito nacional, el Programa Nacional de Inmunizaciones y la notificación obligatoria de enfermedades ${ }^{(40)}$.

\section{El tercer elemento: la vigilancia epidemiológica como tecnología clave de la erradicación}

Según la bibliografía sobre el tema, la tecnología de la aguja bifurcada fue esencial para la erradicación de la viruela, en la medida en que era fácil de manipular, transportar y reutilizar. La vacuna liofilizada también lo fue, pero por su capacidad de resistencia a climas cálidos. La vigilancia epidemiológica no es señalada como una de las tecnologías responsables del fin de la viruela, sino como un producto generado en la experiencia de erradicación. Sin embargo, como muestra el análisis de la bibliografía, los documentos y las entrevistas con los profesionales brasileños de la Campaña, la vigilancia epidemiológica fue el tercer factor clave de la erradicación de la enfermedad en Brasil.

Basada en las acciones de notificación de casos, investigación y vacunación de bloqueo, la vigilancia epidemiológica de la viruela -sea como etapa de la Campaña o como estrategia de vacunación en detrimento de la vacunación masiva- se consolidó a partir de la recolección y sistematización de los datos epidemiológicos de casos de viruela confirmados, o no, a través de exámenes de laboratorio- enviados desde las unidades de vigilancia epidemiológica a las Campañas estatales. Al poseer esos datos, el equipo de médicos de la Campaña conseguía planificar y ejecutar las medidas de vacunación de bloqueo y contenía posibles brotes. En paralelo, el equipo de epidemiología realizaba la tabulación y el estudio de los datos, para especificar las tasas de personas susceptibles a la enfermedad, además de controlar la efectividad de las acciones, tanto de la vacunación masiva como de bloqueo.

Dos de los profesionales que trabajaron en la vigilancia epidemiológica de la Campaña, uno en Rio Grande do Sul, y otro en la Campaña de Bangladesh, analizaron sus experiencias en investigaciones de maestría en salud pública ${ }^{(41,42)}$. En esos trabajos, ambos analizaron los métodos y la organización de la vigilancia epidemiológica de la viruela (y de la poliomielitis), y mostraron cómo el procedimiento fue central para la erradicación de la viruela en Brasil y en los países de África y de Asia. Además, según Verani ${ }^{(42)}$, la investigación epidemiológica de la viruela, como estrategia de acción, habría sustituido la vacunación masiva y permitido que la meta de erradicación pudiera ser alcanzada, a pesar de los casi 200 años de convivencia entre la vacuna antivariólica y la enfermedad.

En esa misma dirección, Chagas ${ }^{(4)}$ señala que, aunque Brasil no haya asumido la vigilancia epidemiológica como estrategia central del combate contra la viruela debido a los compromisos asumidos con la OPS/OMS 
para realizar la Campaña a través de la vacunación masiva, la vigilancia posibilitó alcanzar la erradicación de la enfermedad, hecho que no había sucedido con la Campaña Nacional Contra la Viruela, que usaba solo campañas de vacunación esporádicas, sin ningún mecanismo de control y evaluación de sus acciones. Así, la vigilancia epidemiológica implementada posteriormente por la Campaña de Erradicación de la Viruela, al hacer el seguimiento de la evolución de la tasa de incidencia de casos de viruela y su notoria disminución, conseguía evaluar el impacto del programa de erradicación y garantizar que la enfermedad no volviera a aparecer en las regiones donde ya había sido eliminada.

Más que un importante legado de las actividades de la Campaña, la vigilancia epidemiológica se caracterizó como una herramienta esencial de la propia erradicación de la enfermedad. Incluso, si bien fue pregonada como estrategia de trabajo propuesta por la OMS, la vacunación a partir de la vigilancia no fue adoptada como estrategia por la Campaña nacional, que optó por la vacunación masiva. Sin embargo, en los estados en los que fue aplicada (Paraná, Bahía y Rio Grande do Sul) y en la fase de vigilancia del plan nacional de erradicación, la vigilancia epidemiológica se mostró tan esencial en el combate contra la viruela como la aguja bifurcada y la vacuna liofilizada.

Las acciones de control posterior a la vacunación masiva y la utilización de la estrategia de vacunación de bloqueo solo fueron posibles con el empleo de las técnicas de notificación e investigación implementadas por la vigilancia epidemiológica. Además, al mismo tiempo que las unidades de vigilancia epidemiológica demandaban la actuación de profesionales calificados en sus operaciones, la calificación de estos mismos profesionales posibilitó que estas unidades garantizaran que la erradicación de la viruela se concretara.

La creación del cargo de técnico en epidemiología y la formación de puestos de notificación y de unidades de vigilancia en el ámbito estatal modernizaron y consolidaron las nuevas acciones en el campo de la salud pública en términos institucionales. Al mismo tiempo, posibilitaron que nuevas enfermedades entraran en la agenda de acción del Ministerio de Salud, no en forma de campañas, como había sido hasta entonces ${ }^{(10,12)}$, sino de acciones efectivas de control y actuación, como los días nacionales de vacunación para combatir la poliomielitis y el sarampión.

Además, generaron oportunidades de formación para los antiguos cuadros de salud pública, haciendo posible para algunos de ellos la consolidación de una carrera nacional e internacional en la erradicación de la viruela y de la poliomielitis. Por lo tanto, la erradicación de la viruela significó no solo el control y la eliminación de una enfermedad milenaria, sino también, el rediseño de la salud pública brasileña y de sus instituciones en el nivel nacional.

\section{CONCLUSIONES}

\section{Sobre el redescubrimiento de la viruela: sanitaristas, políticos, intereses nacionales e internacionales}

Si bien en un escenario más general, la erradicación de la viruela se justificó por la existencia de una vacuna eficaz, por el hecho de que el virus no poseía otro reservorio que los seres humanos, y por el ahorro que los países tendrían al no necesitar depender más de sus presupuestos para la vacunación de mantenimiento, en Brasil, la enfermedad no estaba en la agenda de salud pública de la época y por eso es necesario entretejer algunas consideraciones.

Desde las medidas profilácticas de inicios del siglo XX y de las acciones de saneamiento ${ }^{(10,12)}$, la viruela pasó a ocupar un lugar secundario en el plan de salud pública nacional. La concentración en la fiebre amarilla y en la malaria, aunque no hizo desaparecer la práctica de la vacunación antivariólica, llevó a las autoridades de salud a emplear esfuerzos y planes de cooperación para intentar eliminar el mosquito transmisor de esas enfermedades y formular vacunas y otros fármacos eficaces ${ }^{(11)}$. 
Así, con las escasas estadísticas de control de casos de la enfermedad y con el registro de algunas epidemias esporádicas, la viruela, como problema de salud pública, tuvo que ser "redescubierta" para poder disputar un lugar dentro de las acciones de salud comandadas por las autoridades públicas. La Campaña Nacional Contra la Viruela, aunque haya sido planificada a nivel nacional, era de responsabilidad de los poderes locales y, por ese motivo, no tuvo el efecto deseado de eliminar la enfermedad en el territorio nacional, y su ejecución quedó atada a las prioridades locales que, muchas veces, no incluían la viruela como enfermedad a ser combatida.

Como Brasil era el único país del continente que no había erradicado la enfermedad, la presión internacional para la implementación de la Campaña de Erradicación de la Viruela y la ayuda financiera y técnica ofrecida al país por la OPS/OMS contribuyeron a otorgar mayor legitimidad a las acciones. Al mismo tiempo, los pocos datos epidemiológicos disponibles también se movilizaron, a la par del pasado epidémico y del intento frustrado de Oswaldo Cruz de acabar con la enfermedad a inicios del siglo XX.

Así, al "redescubrir" la viruela como problema de salud pública nacional, incluso de forma involuntaria, las autoridades y los profesionales de salud brasileños involucrados en la campaña de erradicación dieron nuevos aires a los servicios de salud, instauraron nuevos protocolos sanitarios, crearon nuevas áreas de acción para los profesionales de la salud y se rehicieron como expertos en un área que siempre fue de punta en la medicina nacional: la salud pública. A su vez, tales profesionales reinventaban la medicina brasileña, al traducir en conocimiento científico sistematizado los conceptos y las prácticas de la epidemiologia, abriendo la oportunidad para el surgimiento de un nuevo profesional de la medicina: el epidemiólogo.

Analizado en retrospectiva, el combate contra la viruela, en el inicio del siglo XX y a partir de la década de 1960, incluso envuelto en controversias políticas y científicas, se desplegó más allá de las medidas profilácticas de control y erradicación de la enfermedad. Estas medidas posibilitaron la construcción y la consolidación de instituciones sanitarias y científicas, además de los protocolos de salud pública, que se ampliaron a inicios del siglo con la creación de las primeras instituciones de investigación del país y, en la década de 1960, con la agrupación y consolidación de organismos estatales en el área de la salud que estaban dispersos entre diferentes ministerios y secretarías.

La crónica de un éxito consolidado nos permite analizar el combate contra la viruela y ver, además de su erradicación, las raíces históricas de las instituciones, los protocolos y las acciones de salud que actualmente operan en el país.

\section{AGRADECIMIENTOS}

El autor agradece a la Fundação de Amparo à Pesquisa do Estado de São Paulo por las becas concedidas (FAPESP 2015/21537-8 y 2016/19402-0) y a la profesora, doctora y directora Heloisa Pontes, por el apoyo. Las opiniones emitidas son de entera responsabilidad de su autor y no representan las opiniones de la agencia de fomento.

\section{REFERENCIAS BIBLIOGRÁFICAS}

1. Carvalho JM. Os bestializados: o Rio de Janeiro e a República que não foi. São Paulo: Companhia das Letras; 2006.

2. Chalhoub S. Cidade febril: cortiços e epidemias na Corte Imperial. São Paulo: Companhia das Letras; 2001. 
3. Sevcenko N. A revolta da vacina: mentes insanas em corpos rebeldes. São Paulo: Cosac Naify; 2010.

4. Chagas DC. Erradicando doenças: De projeto internacional ao sistema de vigilância epidemiológica - A erradicação da varíola no Brasil (1900-1970). [Dissertação de Mestrado]. Rio de Janeiro: Casa de Oswaldo Cruz, Fundação Oswaldo Cruz; 2008.

5. Gazêta $A A B$. Uma contribuição à história do combate à varíola no Brasil: do controle à erradicação. [Teses de Doutorado]. Rio de Janeiro: Casa do Oswaldo Cruz, Fundação Oswaldo Cruz; 2006.

6. Gazêta AAB. A campanha de erradicação da varíola no Brasil. [Dissertação de Mestrado]. Rio de Janeiro: Núcleo de Estudos de Saúde Coletiva, Universidade Federal do Rio de Janeiro; 2001.

7. Hochman G. Priority, invisibility and eradication: The history of smallpox and the Brazilian public health agenda. Medical History. 2009;53(2):229-252.

8. Hochman G. Vacinação, varíola e uma cultura da imunização no Brasil. Ciência \& Saúde Coletiva. 2011;16(2):375-386.

9. Foucault M. Segurança, território e população: curso no Collége de France (1975-1976). São Paulo: Martins Fontes; 2008.

10. Hochman G. A era do saneamento: as bases da política de saúde pública no Brasil. São Paulo: Hucitec, ANPOCS; 1998.

11. Lowy I. Virus, moustiques et modernite: la fievre jaune au Bresil, entre science et politique. Paris: Archives Contemporaines; 2001.

12. Lima NT. Um sertão chamado Brasil: intelectuais e representação geográfica da identidade nacional. Rio de Janeiro: IUPERJ/UCAM, Revan; 1999.

13. Amaral C. Depoimento: acervo de depoimentos orais memória da poliomielite. Rio de Janeiro: Casa de Oswaldo Cruz, Departamento de Arquivo e Documentação; 2001.

14. Amaral C. Entrevista. Niterói: Arquivo pessoal; 2017. 1 arquivo digital: 2h 30min.

15. Brasil. Portaria $n^{\circ} 55$, de 29 de janeiro de 1980: Extingue, a partir desta data, em todo o território nacional, a obrigatoriedade da vacina antivariólica, cuja aplicação ficará restrita as exigências do Regulamento Sanitário Internacional. Diário Oficial da República Federativa do Brasil, Rio de Janeiro; 29 ene 1980. Seção II, p. 1872.
16. Fenner F, Henderson DA, Arita I, Jezek Z, Ladnyi ID. Smallpox and its eradication. Genebra: World Health Organization; 1988.

17. Brasil. Portaria $n^{\circ} 23$, de 18 de janeiro de 1962. Institui a Campanha Nacional Contra a Varíola, com a finalidade de erradicar essa endemia do território nacional. Diário Oficial da República Federativa do Brasil, Rio de Janeiro; 18 ene 1962. Seção I, p. 1097.

18. Risi Jr JB. Depoimento: Projeto vacina antivariólica. Rio de Janeiro: Fundação Oswaldo Cruz, Casa de Oswaldo Cruz; 2005.

19. Sacramento J. Gênero e tecnologia na erradicação da varíola. Revista Gênero. 2018;18(2):80102.

20. Henderson DA. Smallpox: the death of a disease: the inside story of eradicating a worldwide killer. Amherst: Prometheus Books; 2009.

21. Foege $W H$. House on fire: the fight to eradicate smallpox. New York: University of California Press, Milbank Memorial Fund; 2012.

22. Brown TM, Cueto M, Fee E. A transição de saúde pública "internacional" para "global" e a Organização Mundial da Saúde. História, Ciências, Saúde-Manguinhos. 2006;13(3):623-647. doi: $10.1590 /$ S0104-59702006000300005.

23. Cueto M. Saúde global: uma breve história. Rio de Janeiro: Fiocruz; 2015.

24. Sacramento J. Saberes, poderes e corporalidades: a biopolítica da erradicação da varíola. [Dissertação de Mestrado]. Campinas: Instituto de Filosofia e Ciências Humanas, UNICAMP; 2018.

25. Brasil. Lei 59.153, de 31 de agosto de 1966. Institui, no Ministério da Saúde, a Campanha de Erradicação da Varíola e dá outras providências. Diário Oficial da República Federativa do Brasil, Rio de Janeiro; 2 sep 1966. Seção 1, p. 10139.

26. Fischmann A. Depoimento: Projeto vacina antivariólica. Rio de Janeiro: Fundação Oswaldo Cruz, Casa de Oswaldo Cruz; 2005.

27. Silveira CM. Depoimento: Projeto vacina antivariólica. Rio de Janeiro: Fundação Oswaldo Cruz, Casa de Oswaldo Cruz; 2005.

28. São Paulo. Decreto 49.021, de 30 de novembro de 1967: Dispõe sôbre a instituição da "Campanha de Erradicação da Varíola", na Secretaria da Saúde Pública e da Assistência Social, e dá outras providências. Diário Oficial do Estado de São Paulo, São Paulo; 1 dic 1967. 
29. Arita I, Nakane M. Successful eradication of smallpox and the prospect of disease eradication efforts in the twenty-first century. En: Bhattacharya S, Messenger S, (eds.). The global eradication of smallpox. New Delhi: Oriente BlackSwan; 2010.

30. Sanson Y, Tognosi WN, Barcellos M, Passeri G. A enfermagem na Campanha de Erradicação da Varíola: Relatório interno da CEV/SP; 1970.

31. Fernandes TMD, Chagas DC, Souza EM. Varíola e vacina no Brasil no século $X X$ : institucionalização da educação sanitária. Ciência \& Saúde Coletiva. 2011;16(2):479-489. doi: 10.1590/S141381232011000200011.

32. Reinhardt BH. The Global Great Society and the US commitment to smallpox eradication. Endeavour. 2010;34(4):164-172. doi: 10.1016/j.endeavour.2010.09.006.

33. Reinhardt $\mathrm{BH}$. The end of a global pox: American and the eradication of smallpox in the Cold War era. Chapel Hill: The University of North Carolina Press; 2015.

34. Bhattacharya S. Struggling to a monumental triumph: re-assessing the final phases of the smallpox eradication program in India, 1960-1980. História, Ciências, Saúde-Manguinhos. 2007;14 (4):1113-1129. doi: 10.1590/S0104-59702007000 400002.

35. Brilliant L, Conrad CW. The eradication of smallpox from India. En: Bhattacharya S, Messenger S, (eds.). The global eradication of smallpox. New Delhi: Oriente BlackSwan; 2010.

36. Quadros C. Depoimento: Acervo de depoimentos orais memória da poliomielite. Rio de
Janeiro: Fiocruz, Casa de Oswaldo Cruz, Departamento de Pesquisa, Departamento de Arquivo e Documentação; 2001.

37. Quadros C. Depoimento: Projeto vacina antivariólica. Rio de Janeiro: Fundação Oswaldo Cruz, Casa de Oswaldo Cruz; 2005.

38. Fischmann A. Depoimento: Acervo de depoimentos orais memória da poliomielite. Rio de Janeiro: Fiocruz, Casa de Oswaldo Cruz, Departamento de Pesquisa, Departamento de Arquivo e Documentação; 2001.

39. Brasil, Ministério da Saúde, Superintendência de Campanhas de Saúde Pública, Campanha de Erradicação da Varíola. Boletins Semanais da Campanha de Erradicação da Varíola; 1969-1974. Localizado en: Arquivo Histórico, Museu de Saúde Pública Emílio Ribas, São Paulo, Brasil.

40. Brasil. Lei 6.259 , de 30 de outubro de 1975 : Dispõe sobre a organização das ações de Vigilância Epidemiológica, sobre o Programa Nacional de Imunizações, estabelece normas relativas à notificação compulsória de doenças, e dá outras providências. Diário Oficial da República Federativa do Brasil, Brasília; 31 oct 1975. Seção 1, p. 14473.

41. Fischmann A. Investigação epidemiológica de varíola no estado do Rio Grande do Sul, Brasil. [Dissertação de Mestrado]. São Paulo: Faculdade de Saúde Pública, Universidade de São Paulo; 1978.

42. Verani JFS. A vigilância epidemiológica na erradicação de doenças: A varíola e a poliomielite. [Dissertação de Mestrado]. Rio de Janeiro: Escola Nacional de Saúde Pública, Fundação Oswaldo Cruz; 1990.

\section{FORMA DE CITAR}

Sacramento J. Crónica de un éxito consolidado: las políticas de erradicación de la viruela en Brasil (1962-1973). Salud Colectiva. 2019;15:e2167. doi: 10.18294/sc.2019.2167.

Recibido: 7 de febrero de 2019 | Versión final: 13 de julio de 2019 | Aprobado: 16 de julio de 2019

Esta obra está bajo una licencia de Creative Commons Reconocimiento-NoComercial 4.0

Internacional. Reconocimiento - Permite copiar, distribuir y comunicar públicamente la obra.

A cambio, se debe reconocer y citar al autor original. No Comercial — Esta obra no puede ser

utilizada con finalidades comerciales, a menos que se obtenga el permiso.

http://dx.doi.org/10.18294/sc.2019.2167

Este artículo fue traducido del portugués por Viviana Martinovich. 